\title{
BMJ Open Identification of new biomarkers to promote personalised treatment of patients with inflammatory rheumatic disease: protocol for an open cohort study
}

\author{
Tina Marie Kringelbach, ${ }^{1,2}$ Bente Glintborg, ${ }^{1,3,4}$ Estrid V Hogdall, ${ }^{1,2,5}$ \\ Julia Sidenius Johansen, ${ }^{1,5,6,7}$ Merete Lund Hetland, ${ }^{1,3,4,5}$ on behalf of The \\ Biomarker Protocol Study Group
}

To cite: Kringelbach TM, Glintborg B, Hogdall EV, et al. Identification of new biomarkers to promote personalised treatment of patients with inflammatory rheumatic disease: protocol for an open cohort study. BMJ Open 2018;8:e019325. doi:10.1136/ bmjopen-2017-019325

- Prepublication history for this paper is available online. To view these files, please visit the journal online (http://dx.doi. org/10.1136/bmjopen-2017019325).

TMK and BG contributed equally.

Received 26 August 2017 Revised 13 November 2017 Accepted 4 December 2017

Check for updates

For numbered affiliations see end of article.

Correspondence to Dr Merete Lund Hetland; merete.hetland@dadlnet.dk

\section{ABSTRACT}

Introduction The introduction of biological diseasemodifying antirheumatic drugs (bDMARDs) has improved the treatment of inflammatory rheumatic diseases dramatically. However, bDMARD treatment failure occurs in $30 \%-40 \%$ of patients due to lack of effect or adverse events, and the tools to predict treatment outcomes in individual patients are currently limited. The objective of the present study is to identify diagnostic, prognostic and predictive biomarkers, which can be used to (1) diagnose inflammatory rheumatic diseases early in the disease course with high sensitivity and specificity, (2) improve prognostication or (3) predict and monitor treatment effectiveness and tolerability for the individual patient. Methods and analysis The present study is an observational and translational open cohort study with prospective collection of clinical data and biological materials (primarily blood) in patients with inflammatory rheumatic diseases treated in routine care. Patients contribute with one cross-sectional blood sample and/ or are enrolled for longitudinal follow-up on initiation of a new DMARD (blood sampling after 0, 3, 6, 12, 24, 36, 48, 60 months of treatment). Other biological materials will be collected when accessible and relevant. Demographics, disease characteristics, comorbidities and lifestyle factors are registered at inclusion; DMARD treatment and outcomes are collected repeatedly during follow-up. Currently (July 2017), $>5000$ samples from approximately 3000 patients have been collected. Data will be analysed using appropriate statistical analyses.

Ethics and dissemination The protocol is approved by the Danish Ethics Committee and the Danish Data Protection Agency. Participants give written and oral informed consent. Biomarkers will be evaluated and published according to the Reporting Recommendations for Tumour Marker (REMARK) prognostic studies,

Strengthening the Reporting of Observational Studies in Epidemiology (STROBE) and the Standards for Reporting of Diagnostic Accuracy (STARD) guidelines. Results will be published in peer-reviewed scientific journals and presented at international conferences.

Trial registration number NCT03214263.
Strengths and limitations of this study

- Nationwide collection of biological materials and corresponding extensive clinical data provides the opportunity to discover and/or validate a wide range of diagnostic, prognostic and predictive biomarkers in patients with inflammatory rheumatic disease.

- Recruitment of patients treated in routine care is expected to provide valuable data on 'real-life patients' (eg, elderly patients with comorbidities), which are different from the more homogeneous patient population in randomised controlled trials.

- Standardised collection of samples and quality control ensures comparability between samples from different departments, and enables research in less common rheumatic diseases.

- Patient recruitment and follow-up in routine care and across several rheumatic diagnoses and treatments will be associated with some limitations in clinical and biological data.

- The non-randomised study design inherits a risk of confounding and thorough statistical analysis and confounder adjustment is therefore important.

\section{INTRODUCTION}

Rheumatoid arthritis (RA), psoriatic arthritis (PsA) and axial spondyloarthritis (AxSpA) are examples of chronic inflammatory rheumatic diseases characterised by pain, disability and progressive decrease in workability, and are associated with comorbidity and risk of early death. ${ }^{12}$ The impact of these chronic diseases on the patients should be minimised through early diagnosis followed by targeted therapy with minimal side effects. If and when remission is achieved, the patient has the potential to maintain a life with few restrictions-a desirable outcome both for the patient and for society. 
The medical treatment of inflammatory rheumatic diseases has improved dramatically during the last decades. This is mainly due to an increased acknowledgement of the treat-to-target concept with conventional synthetic disease-modifying antirheumatic drugs (csDMARDs) in RA, which implies strict monitoring and aggressive treatment strategies with add-on or switching of therapy according to clinical response or side effects. ${ }^{3-5}$ Furthermore, the biological DMARDs (bDMARDs), for example, tumour necrosis factor alpha inhibitors or other specific modulators of inflammatory signal transduction, have improved outcomes for patients otherwise refractory to treatment with csDMARDs. ${ }^{4}$ New treatment modalities including targeted synthetic DMARDs (tsDMARDS, eg, Janus kinase (JAK) inhibitors) are being introduced, and the first biosimilar bDMARDs have been marketed. bDMARDs and JAK inhibitors are expensive, and treatment failure, defined as lack of effect or serious adverse events, occurs in $30 \%-40 \%$ of patients treated with bDMARDs. ${ }^{67}$ Tools to predict treatment outcomes and side effects in the individual patient are currently limited. $^{8}$

In 1998, the National Institutes of Health Biomarkers Definitions Working Group defined a biomarker as 'a characteristic that is objectively measured and evaluated as an indicator of normal biological processes, pathogenic processes or pharmacological responses to a therapeutic intervention'. ${ }^{10}$ Biomarkers are divided in three categories: (1) diagnostic biomarkers, which may be used for early diagnosis of a given disease. ${ }^{11}{ }^{12}$ The ideal diagnostic biomarker should establish the correct diagnosis with high sensitivity and specificity; (2) prognostic biomarkers, which correlate with specific clinical outcomes, and thus progression of disease, regardless of any treatment and (3) predictive biomarkers, which may be used to predict whether a given patient may benefit from a given treatment. ${ }^{1314}$ Hence, biomarkers may be promising tools to personalise the treatment of patients with inflammatory rheumatic disease. Biomarkers in blood and tissue include a wide range of molecules with different characteristics such as DNA, RNA, microRNA (miRNA), proteins and metabolites. Genetic variation can be caused by single nucleotide polymorphisms (SNPs), nucleotide insertions/deletions and copy number variations, among which the SNP and insertions/deletions are the most common types of genetic variation. ${ }^{15-19}$ miRNAs are small single-stranded, endogenous, non-coding RNAs (18-25 nucleotides) and play essential roles in regulating gene expression, cell development, differentiation and proliferation. ${ }^{19-21}$ The human proteome constitutes all expressed human proteins and reflects the biological activity of the patient, and proteomics is increasingly used to investigate treatment response ${ }^{22} 23$ or to stratify responding versus non-responding patients. ${ }^{24} 25$ The metabolome is defined as the complete set of metabolites $<1500 \mathrm{Da}$ found in a given biological sample. It is a dynamic entity, which reflects the interaction between the individual genetic background and factors such as pathophysiological conditions, diet and pharmacological treatment. ${ }^{26}$ In patients with RA, PsA and AxSpA, these biomarkers may be related to the disease itself, the associated inflammation or treatment-related pharmacokinetics. Biomarkers can be detected in peripheral blood, synovial fluid, circulating cells or cell-free DNA in plasma or in tissue (eg, cartilage, bone and synovial membrane).

Currently, some biomarkers are used as part of the classification of arthritis patients, for example, IgM rheumatoid factor (IgM-RF), cyclic citrullinated protein antibodies (anti-CCP), C reactive protein (CRP) and human leucocyte antigen B27 (HLA-B27) ${ }^{27-29}$ However, for individual patients, these few biomarkers cannot differentiate a patient from a healthy subject with high specificity or predict mild versus severe disease. Radiographical imaging is used routinely to assess cumulated joint damage;however, biomarkers have the potential of being a more feasible, specific and reproducible tool for both diagnostic and prognostic purposes and for the monitoring of treatment and disease progression.

The present protocol is an observational, prospective, translational research study of patients with rheumatologic disease followed in the nationwide Danish DANBIO Registry $^{30}$ and the Danish Rheumatologic Biobank. ${ }^{31} 32$ The objective is to identify new diagnostic, prognostic and predictive biomarkers, which can be used to (1) diagnose inflammatory rheumatic diseases early in the disease course with high sensitivity and specificity, (2) predict patient prognosis regardless of treatment or (3) predict and monitor the effective treatment for the individual patient with minimised risk of side effects. This protocol has been prepared and presented according to the Reporting Recommendations for Tumour Marker (REMARK) prognostic studies and Strengthening the reporting of Observational Studies in Epidemiology (STROBE) guidelines. ${ }^{13} 33$

\section{MATERIALS AND METHODS}

\section{Study design and setting}

Biological samples and clinical data are collected prospectively in patients with rheumatic disease treated in routine care. Clinical data and outcomes are registered in the Danish nationwide quality registry DANBIO, ${ }^{30} 34$ and biological samples are collected via the Danish Rheumatologic Biobank. ${ }^{31} 32$ Patient inclusion started in May 2015 and will continue until 2025 with follow-up until 2030. If needed, the inclusion period can be expanded.

DANBIO is a nationwide, Danish register which serves as a clinical database for monitoring of clinical quality of treatment and which may be used for research purposes. DANBIO was established in year 2000 and data collection occurs prospectively by a web-based system used in routine care at Danish hospital Departments of Rheumatology and in primary care (private practising specialists of rheumatology). It is mandatory to monitor patients with inflammatory rheumatic diseases treated with bDMARDs and patients with newly diagnosed RA irrespective of 
A Cross-sectional samples

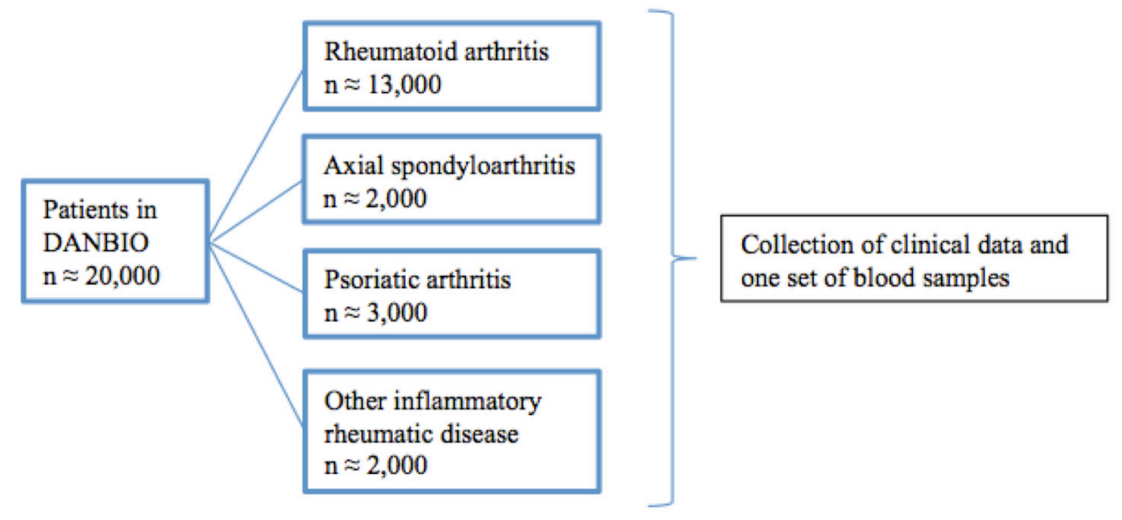

B Longitudinal samples

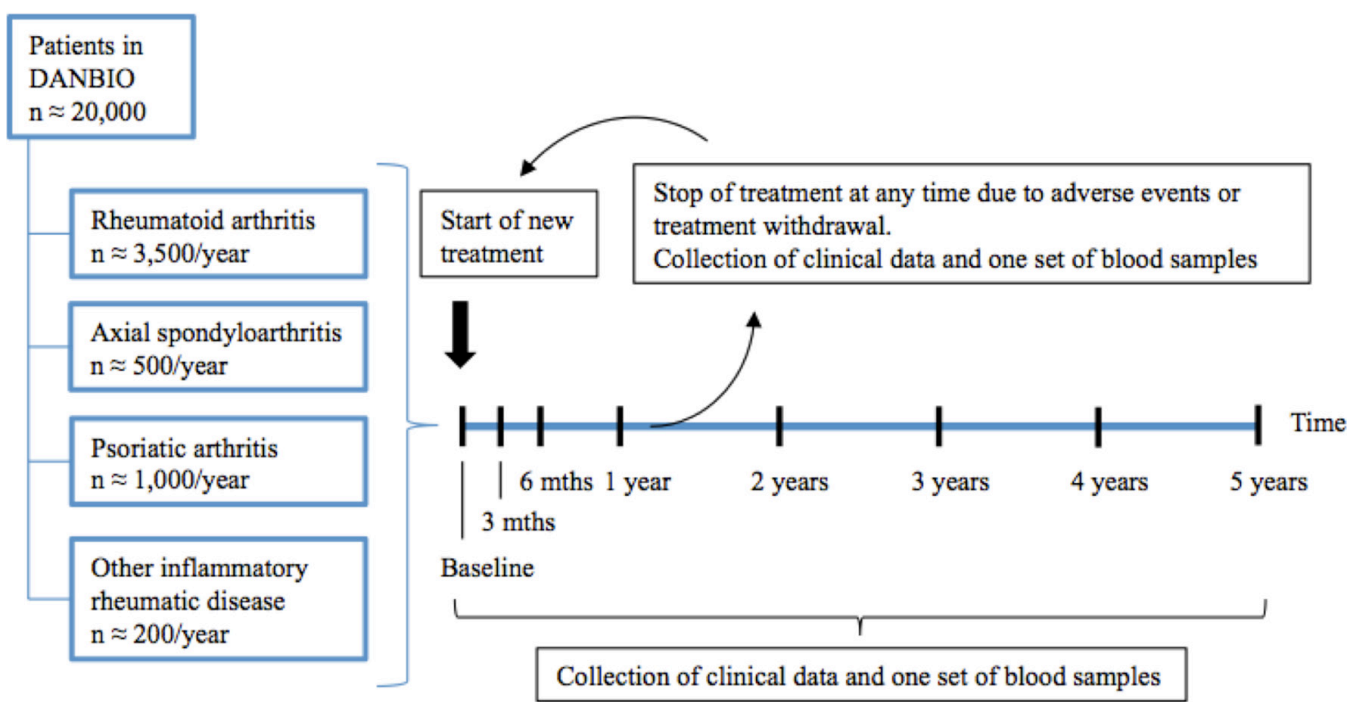

Figure 1 Schematic presentation of study design and sampling strategies. Any patient diagnosed with RA, AxSpA, PsA, other inflammatory rheumatic disease or tissue disorder, or suspected for one of these, may participate when they meet for a scheduled routine clinical visit. These patients can provide one cross-sectional blood sample (A) or may be included for longitudinal follow-up (B) when they start treatment with a new DMARD (see text). See figure 2 for details on blood handling and storage. Numbers $(n)$ indicate patients potentially eligible for inclusion in one or more of the study arms at the time of protocol approval. By 1 January 2018, the number of patients in DANBIO is approximately 50,000. AxSpA, axial spondyloarthritis; DMARD, disease-modifying antirheumatic drug; PsA, psoriatic arthritis; RA, rheumatoid arthritis.

treatment. ${ }^{34}$ Data registered in DANBIO are listed in the 'Clinical data' section below. DANBIO represents an excellent tool for monitoring patients in routine care and for research purposes.

The Danish Rheumatologic Biobank was established in 2015 through nationwide collaboration between Departments of Rheumatology and Departments of Clinical Biochemistry in Denmark. The Danish Rheumatologic Biobank is organised according to the infrastructure of the well-established Danish CancerBiobank, ${ }^{31}$ and both biobanks are part of the Bio- and Genome Bank Denmark funded by Danish Regions (the governmental organisation who runs the public hospitals in Denmark). The foundation of the Danish Rheumatologic Biobank was funded by the Danish Rheumatism Association and Danish Regions. By 1 June 2017, 12 hospitals from all parts of Denmark (Rigshospitalet; North Denmark
Regional Hospital; King Christian 10th Hospital for Rheumatic Diseases, Graasten; Aarhus University Hospital; Copenhagen University Hospital, Gentofte; Zealand University Hospital, Køge; OUH Svendborg Hospital; Odense University Hospital; Aalborg University Hospital; Hospital Lillebaelt, Vejle; Randers Regional Hospital and University Hospital Bispebjerg and Frederiksberg) participated in the Danish Rheumatologic Biobank, and additional hospitals are continuously joining. Different types of biological material (eg, blood, tissue, synovial fluid and urine) are collected, handled and stored according to nationally approved standard operating procedures $(\mathrm{SOPs})^{31}$ (see Biological samples section).

Patients contribute primarily with blood samples, but also other types of biological materials (synovial fluid and surgical tissue), when these are accessible and relevant. Patients contribute with one or more of the following 
samples: (1) cross-sectional blood samples: patients provide one cross-sectional sample when they meet for a scheduled routine clinical visit (figure 1A); (2) longitudinal blood samples: patients may be enrolled for longitudinal follow-up when they start treatment with a new DMARD. Switching from csDMARD to bDMARD or from one bDMARD to another bDMARD, indicates a new baseline sample (figure 1B) and (3) other biological materials: patients may contribute with representative samples of biological material if they are scheduled for joint puncture with aspiration (synovial fluid), surgery or biopsies (synovia, cartilage, bone, bone marrow or other tissues). Cross-sectional sampling may be done at any given disease stage and at any time point during treatment. Longitudinal blood samples are collected at baseline and after 3 , $6,12,24,36,48$ and 60 months of treatment. In case of serious adverse events or treatment withdrawal, additional blood sampling is performed (figure 1B). Approximately half of the material will be used for the present study. The other half can be made available to other researchers, who wish to cooperate, according to guidelines in the Bioand Genome Bank Denmark.

The present protocol is designed to investigate a broad range of biomarkers in patients with inflammatory rheumatic diseases. One of the first longitudinal cohorts in the study included patients who switched from originator infliximab (IFX, Remicade) to biosimilar IFX (CT-P13, Remsima). According to national guidelines issued in 2015, all Danish patients diagnosed with inflammatory rheumatic diseases (RA, PsA or AxSpA), and treated with originator IFX, were switched to CT-P13. ${ }^{35}$ In association with this non-medical switch, the aim was to investigate the following biomarkers and clinical outcome: (1) effects of the switch on serum IFX (sIFX) and presence of antidrug antibodies (ADAb) and (2) association between sIFX and ADAb at the time of switch on adherence to CT-P13 treatment. ${ }^{36}$ Clinical data and longitudinal blood samples were collected as described in the present protocol.

\section{Participants}

The Biomarker Protocol is an open cohort study, that is, participants may enter and leave the population at different time points during monitoring. Patients are eligible for inclusion if they are followed in routine care and monitored in DANBIO with one of the following diagnoses: RA, AxSpA, PsA, other inflammatory rheumatic diseases or tissue disorders, or are suspected for one of the above. Patients must be able to give written and oral informed consent and be aged $\geq 18$ years. There are no exclusion criteria. Patient inclusion and follow-up will be performed by nurses and physicians when the patients meet for scheduled routine clinical visits. The number of potentially eligible patients for the study is shown in figure 1.

\section{Clinical data}

At the time of inclusion, the following clinical data are collected in DANBIO ${ }^{3034}$ :
1. Patient demographics: for example, age, gender, body weight, diagnosis and disease duration.

2. Exposures: that is, previous and current treatment with corticosteroids, non-steroid anti-inflammatory drugs and DMARDs, including dosing schedule, start and stop date and reason for treatment withdrawal.

3. Outcomes: patient-reported outcomes (eg, Visual Analogue Scales for pain, fatigue, patient's global, Health Assessment Questionnaire, quality of life), Disease Activity Score 28-joints, serum CRP concentration, radiographical status (for RA: erosions on X-rays of hand or feet), and bone mineral density (BMD). In axial disease: Bath Ankylosing Spondylitis (BAS) scores for Disease Activity Index (BASDAI), Function Index (BASFI) and Metrology Index (BASMI) are registered.

4. Comorbidities and lifestyle factors: serum cholesterol, diabetes, blood pressure, cardiovascular disease or other comorbidities, smoking status and exercise habits.

On every new collection of biological material, exposure and outcome data are re-evaluated and registered within 30 days before/after the collection of biological material. Any prescription of medical treatment and the monitoring of disease status (radiographical status, BMD, etc) are done as part of routine care and do not follow a specific study protocol. Data registration in the DANBIO Registry follows DANBIO guidelines. ${ }^{34} 37$

\section{Biological samples}

The collected biological material is primarily blood. Synovial fluid, tissue, cartilage, bone and bone marrow may also be collected, when accessible and relevant. Peripheral blood is collected in one EDTA tube $(9 \mathrm{~mL})$, two serum tubes $(2 \times 9 \mathrm{~mL})$ and one PAXgene blood RNA tube (2.5 mL, Becton and Dickinson, Lyngby, Denmark). Blood samples are processed according to the nationally approved SOP for blood (figure 2 ).$^{31}$ In brief, EDTA whole blood $(1.5 \mathrm{~mL})$ is isolated followed by the centrifugation of EDTA and serum tubes at $2000 \mathrm{xg}$ and $4^{\circ} \mathrm{C}$ for $10 \mathrm{~min}$. After centrifugation $2 \times 2 \mathrm{~mL}$ EDTA plasma, $1 \times$ EDTA buffy coat and $4 \times 2 \mathrm{~mL}$ serum are isolated. PAXgene blood RNA tubes are kept at room temperature for 2-72 hours, hereafter frozen at $-20^{\circ} \mathrm{C}$ for $24-72$ hours, and finally stored long term at $-80^{\circ} \mathrm{C}$. Whole blood and buffy coat are stored at $\leq-20^{\circ} \mathrm{C}$; plasma and serum are stored at $-80^{\circ} \mathrm{C}$.

Synovial fluid is collected in EDTA tubes $(9 \mathrm{~mL})$ and centrifuged at $2000 \times \mathrm{g}$ and $4^{\circ} \mathrm{C}$ for $10 \mathrm{~min}$. The cell-free supernatant is transferred to $5 \mathrm{~mL}$ cryotubes and each cell pellet is resuspended in $1 \mathrm{~mL}$ supernatant and pooled in $5 \mathrm{~mL}$ cryotubes. Sample processing results in: $\leq 20 \times 5 \mathrm{~mL}$ cell-free synovial fluid and $\leq 2 \times 5 \mathrm{~mL}$ cell pellet, which are stored long term at $-80^{\circ} \mathrm{C}$.

Preanalytical factors such as date and time of sampling, handling and storage, temperature during transportation, and the exact handling procedure are registered in 


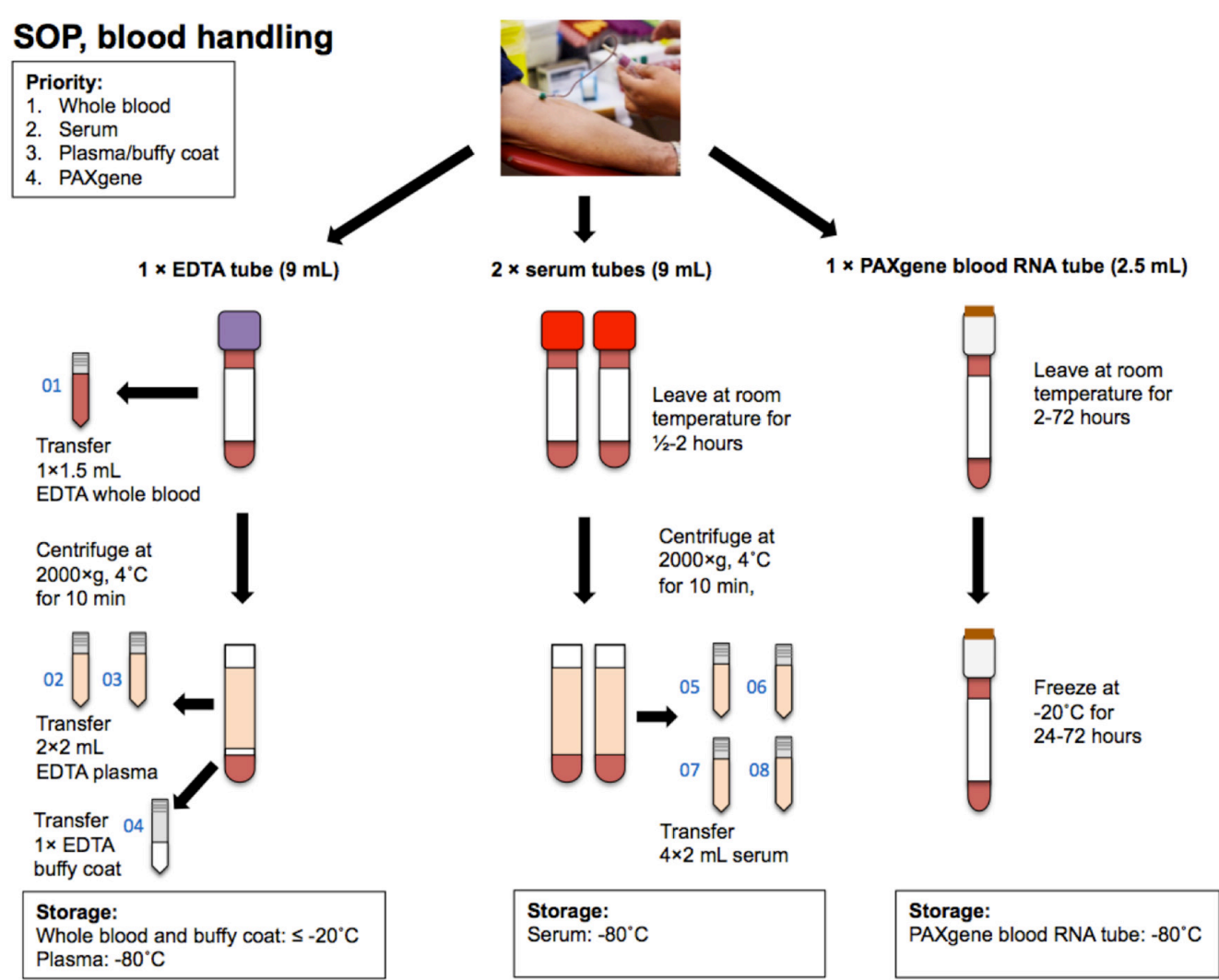

Figure 2 Standard operating procedure (SOP) for blood handling in the Danish Rheumatologic Biobank. ${ }^{31}$ Peripheral blood is collected in one EDTA tube, two serum tubes and one PAXgene blood RNA tube. Serum tubes coagulate at room temperature for $30 \mathrm{~min}$ to 2 hours. From the EDTA tube, $1.5 \mathrm{~mL}$ whole blood is isolated. EDTA and serum tubes are centrifuged at $2000 \times \mathrm{xg}$ and $4^{\circ} \mathrm{C}$ for $10 \mathrm{~min}$. EDTA plasma $(2 \times 2 \mathrm{~mL})$, EDTA buffy coat and serum $(4 \times 2 \mathrm{~mL})$ are isolated. Processed blood samples are stored at $\leq-20^{\circ} \mathrm{C}$. PAXgene blood RNA tubes are kept at room temperature for $2-72$ hours, then frozen at $-20^{\circ} \mathrm{C}$ for $24-72 \mathrm{hours}$ and stored at $-80^{\circ} \mathrm{C}$.

the nationwide Bio- and Genome Bank Denmark registry. All samples are pseudonymised before storage.

\section{Assay methods}

The protocol aims to investigate the following biomarkers in blood, synovial fluid or tissue:

1. Genetic variation using next-generation sequencing and whole-genome sequencing, and RNA and miRNA expression profiles.

2. Protein biomarker profiles of inflammation, and bone and cartilage metabolism using, for example, the MultiBiomarker Disease Activity score (a panel of 12 proteins) ${ }^{38}$ (Crescendo Bioscience, South San Francisco, California, USA), Proseek Multiplex protein arrays (panels of 92 proteins) (Olink Proteomics, Uppsala, Sweden, www.olink.com) or proteomics platforms, such as mass spectrometry, protein arrays or multiplexed ELISA.

3. Metabolites using Nuclear Magnetic Resonance-spectroscopy.

4. ADAb against bDMARD and drug concentrations (eg, IFX) using a target-based assay fully automated on the AutoDELFIA (PerkinElmer, Waltham, Massachusetts, USA) immunoassay platform (Oslo University Hospital, Radiumhospitalet, Oslo, Norway).
All samples will be analysed in pseudonymised form to ensure blinded testing by the laboratory personnel. The list of specific diagnostic, prognostic and predictive biomarkers will be updated continuously according to new discoveries. The methods for biomarker analysis are rapidly expanding and improving, and the best available method will be used at time of analysis.

\section{Statistical methods}

For the longitudinal samples, it is expected that the numbers collected during a 10-year period will provide sufficient statistical power to identify prognostic and predictive biomarkers if these are present among $>10 \%$ of the patients. Knowledge within the field is still insufficient, thus, it is not possible to perform a comprehensive power calculation; this will, however, be performed before any biomarker analysis is done.

In general, statistical analyses will be done according to available data; the following statistical tests may be used (the list is not complete): comparison of group demographics will be done with Student's t-test, Pearson's $\chi^{2}$ test or Mann-Whitney U test according to the distribution of data. Due to the large size of the dataset the probability for type II error in testing the hypothesis will be low. Treatment duration and time to event can be explored 
with Kaplan-Meier curves, log-rank statistics and Cox regression analyses. Treatment outcomes across groups or according to specific biomarkers will be analysed with logistic regression analyses. Multivariable analyses will be performed in order to study the impact of potential confounders. These confounders may be identified in the DANBIO Registry (gender, age, smoking status or other baseline characteristics). All included patients are recruited and treated in routine care across Denmark and this will inevitably lead to some missing data (missing sampling of biological material, missing registration of corresponding clinical data, whenever biological material is collected, patient lost to follow-up, etc). For sensitivity, various statistical methods may be applied in order to test the robustness of the results. This may be done as last observation carried forward in case of lacking data on clinical outcomes, non-responder imputation or statistical multiple imputation of missing data. Statistical expertise will be included when necessary.

\section{ETHICS AND DISSEMINATION}

The Protocol is approved by the Danish Ethics Committee (H-2-2014-086, supplementary protocol 49419) and the Danish Data Protection Agency (RH-2015-297, I-suite 04318). The Danish Rheumatologic Biobank is approved by the Danish Data Protection Agency (GLO-2015-6, I-Suite 03490). All patients receive verbal and written information before enrolment, and give oral and written consent at baseline according to the guidelines from the Danish Ethics Committee. All patients are informed that they can withdraw from the study at any time without it having consequences for their treatment. In case of withdrawal, samples are discarded and all patient-related registrations deleted from the Bio- and Genome Bank Denmark registry.

The sampled volume of blood for the study is $26.5 \mathrm{~mL}$ per patient-visit and maximum $240 \mathrm{~mL} /$ year. The sampling of blood for the study is performed simultaneously with scheduled routine blood sampling, thus minimising the discomfort for the patient. Synovial fluid, surgical tissue or bone marrow will only be collected if relevant interventions occur as part of routine care and surplus material, not used for diagnostic or therapeutic purposes, is available. The patients will be contacted and informed regarding the overall study results if they indicate interest in this in the patient study consent form. Direct feedback to the patient may be relevant in case of the discovery of mutations in known diseaselinked genes or as random discoveries, and will occur according to the guidelines directed by the Danish Ethics Committee (document no: 1293688, October 2013). The physician in charge of the project at the individual department is responsible for conducting the study in accordance with the Declaration of Helsinki. Study participation does not affect the treatment course of individual patients and the patients will be treated according to clinical practise.
Due to the large number of included patients, it will be possible to perform exploratory as well as validation biomarker studies. We plan to evaluate and publish study results according to the REMARK, ${ }^{13}$ STROBE $^{33}$ and the Standards for Reporting of Diagnostic Accuracy $(\text { STARD })^{39}$ guidelines. Results will be published in international and peer-reviewed scientific journals and presented at international conferences. Negative, positive as well as inconclusive results will be published. If relevant, collaborations with international researchers will be established to facilitate the right expertise for biomarker analyses. The first results (measurements of sIFX and $\mathrm{ADAb}$ drug levels up to 1 year after switch from originator to biosimilar IFX) have been presented 363740

\section{STUDY STATUS}

Patient recruitment started in May 2015 and is expected to continue until 1 January 2025, with follow-up until 1 January 2030. Currently, approximately 3000 patients have been enrolled in the study and $>5000$ blood samples have been collected.

\section{DISCUSSION AND POTENTIAL LIMITATIONS}

In this observational, prospective and translational biomarker study of patients with inflammatory rheumatic diseases, blood samples are collected in routine care and closely linked to extensive clinical data regarding rheumatic disease status and activity, medical treatment, treatment efficacy and adverse events, and comorbidities. The study protocol allows for a large-scale collection of blood and other biological materials with the aim to identify new biomarkers that can be used for improved personalised treatment of patients with inflammatory rheumatic diseases. Additionally, the nationwide collection of biological materials and clinical data is intended to further promote research collaboration within inflammatory rheumatic diseases, both nationally and internationally, in order to ensure research of the highest quality for the benefit of the patients.

Positivity for IgM-RF and anti-CCP are established risk factors for development of RA, and they are currently used as part of classification criteria and as prognostic markers. ${ }^{3}$ In AxSpA, HLA-B27 is part of the disease classification. ${ }^{41}$ Apart from the erythrocyte sedimentation rate and serum-CRP level, no biomarkers are used in routine care, and they cannot predict treatment responses or side effects. The wide range of currently available and future bDMARDs with different modes of action for the treatment of inflammatory arthritis, and the recent introduction of biosimilars and tsDMARDS, stresses the importance of improved ability to select the most effective treatment in the individual patient. Development of diagnostic, prognostic and predictive biomarkers will benefit the treatment of future patients and facilitate personalised medicine. 
Patient recruitment and follow-up in routine care will lead to some limitations in clinical and biological data. Since patients are recruited across several rheumatic diagnoses and treatments, patient inclusion may take some time in order to obtain enough samples for a specific research question. However, since it is mandatory to register patients receiving biological treatment in DANBIO, coverage is high $(\approx 96 \%)^{42}$ and the risk of selection bias low. The risks for the patient are minimal and are out-weighted by the benefits for future patients. The non-randomised study design inherits the risks of confounding, and thorough statistical analysis and confounder adjustment is therefore important. On the other hand, the wide recruitment of patients treated in routine care may provide valuable data on, for example, elderly patients with comorbidities. This may be a valuable supplement to data generated in randomised trials.

Hopefully, the results of the present study will provide us with new biomarkers that will improve our ability to (1) diagnose rheumatic diseases more accurately and at an earlier stage, (2) prognosticate the development of rheumatic diseases and (3) predict and monitor treatment effectiveness in the individual patient (personalised treatment).

The Danish Rheumatologic Biobank provides an infrastructure for national and international research collaboration. Thus, researchers, who are interested in collaboration regarding samples and/or clinical data from DANBIO should contact the Danish Rheumatologic Biobank ${ }^{31}$ and DANBIO, ${ }^{30}$ respectively.

\section{Author affiliations}

${ }^{1}$ The Danish Rheumatologic Biobank, Capital Region, Denmark

${ }^{2}$ Bio- and Genome Bank Denmark, The Molecular Unit, Department of Pathology, Copenhagen University Hospital Herlev, Herlev, Denmark

${ }^{3}$ Copenhagen Centre for Arthritis Research, Center for Rheumatology and Spine Diseases, Rigshospitalet, Glostrup, Denmark

${ }^{4}$ The Danish DANBIO Registry, Rigshospitalet, Glostrup, Denmark

${ }^{5}$ Department of Clinical Medicine, Faculty of Health and Medical Sciences,

University of Copenhagen, Copenhagen, Denmark

${ }^{6}$ Department of Medicine, Copenhagen University Hospital Herlev, Herlev, Denmark ${ }^{7}$ Department of Oncology, Copenhagen University Hospital Herlev, Herlev, Denamrk

Acknowledgements The Danish Rheumatologic Biobank is acknowledged for handling and storage of biological material. Lab technician Teresa Rozenfeld and IT specialist Niels Steen Krogh are acknowledged for their assistance. The Biomarker Protocol Study Group is acknowledged for their contribution to data registration or sample collection and for review of the present manuscript.

Collaborators Members of the Biomarker Protocol Study Group include the following: Asta Linauskas and Gitte Meyer Larsen (North Denmark Regional Hospital); Anne Gitte Loft, Charlotte Modin and Karin Skovgaard (Aarhus University Hospital); Inge Juul Sørensen and Britt Corfixen (Rigshospitalet); Oliver Hendricks and Charlotte Drachmann (King Christian (10th Hospital for Rheumatic Diseases, Graasten); Hanne Lindegaard and Marina Bjørling-Poulsen (Odense University Hospital); Lene Dreyer (Copenhagen University Hospital, Gentofte); Johnny Raun (Hospital Lillebaelt, Kolding); Mikkel Østergaard (Rigshospitalet); Kim HørslevPetersen (King Christian 10th Hospital for Rheumatic Diseases, Graasten); Henning Bliddal (Frederiksberg Hospital); Claus Rasmussen (North Denmark Regional Hospital); Torkell Ellingsen (Odense University Hospital); Søren Jacobsen (Rigshospitalet); Tove Lorenzen (Silkeborg Regional Hospital); Inger Marie Jensen Hansen (OUH Svendborg Hospital); Annette Schlemmer and Salome Kristensen (Aalborg University Hospital); Kristian Stengaard-Pedersen (Aarhus University Hospital); Michael Stoltenberg and Henrik Nordin (Zealand University Hospital,
Køge); Peter Mosborg (Randers Regional Hospital); Susanne Juhl Pedersen and Christoffer Tandrup Nielsen (Rigshospitalet); Ellen-Margrethe Hauge and Bent Deleuran (Aarhus University Hospital); Cecilie Brahe (Rigshospitalet); Christian Gytz Ammitzbøll (Aarhus University Hospital); Tue Wenzel Kragstrup (Aarhus University); Karen Carlsen (North Zealand University Hospital, Hillerød); Niklas Rye Jørgensen (Rigshospitalet); Dorte Vendelboe Jensen (Copenhagen University Hospital, Gentofte); Heidi Lausten Munk (Odense University Hospital); Michael Kruse Meyer (North Denmark Regional Hospital); Tine Lottenburger (Hospital Lillebaelt, Vejle).

Contributors MLH and JJ wrote the protocol for research ethics committee. MLH, $\mathrm{JJ}, \mathrm{EVH}, \mathrm{TMK}$ and BG contributed to study concept. TMK and BG drafted and revised the manuscript after feedback from all authors. All authors contributed to review of the present manuscript and approved the final version of the manuscript.

Funding The Danish Rheumatism Association and Danish Regions funded the establishment of The Danish Rheumatologic Biobank. Danish Regions are funding the continued collection of biological samples and clinical data. Biomarker analyses will be funded by research grants.

Competing interests None declared.

Patient consent Obtained.

Ethics approval The protocol is approved by the Danish Ethics Committee (H-2-2014-086,supplementary protocol 49419) and the Danish Data Protection Agency (RH-2015-297, I-Suite04318).

Provenance and peer review Not commissioned; externally peer reviewed.

Open Access This is an Open Access article distributed in accordance with the Creative Commons Attribution Non Commercial (CC BY-NC 4.0) license, which permits others to distribute, remix, adapt, build upon this work non-commercially, and license their derivative works on different terms, provided the original work is properly cited and the use is non-commercial. See: http://creativecommons.org/ licenses/by-nc/4.0/

(c) Article author(s) (or their employer(s) unless otherwise stated in the text of the article) 2018. All rights reserved. No commercial use is permitted unless otherwise expressly granted.

\section{REFERENCES}

1. Eriksson JK, Johansson K, Askling J, et al. Costs for hospital care, drugs and lost work days in incident and prevalent rheumatoid arthritis: how large, and how are they distributed? Ann Rheum Dis 2015;74:648-54.

2. Kiadaliri AA, Felson DT, Neogi T, et al. Brief Report: rheumatoid arthritis as the underlying cause of death in thirty-one countries, 1987-2011: trend analysis of World Health Organization mortality database. Arthritis Rheumatol 2017;69:1560-5.

3. Smolen JS, Landewé R, Bijlsma J, et al. EULAR recommendations for the management of rheumatoid arthritis with synthetic and biological disease-modifying antirheumatic drugs: 2016 update. Ann Rheum Dis 2017;76:960-77.

4. Smolen JS, Breedveld FC, Burmester GR, et al. Treating rheumatoid arthritis to target: 2014 update of the recommendations of an international task force. Ann Rheum Dis 2016;75:3-15.

5. Gossec L, Smolen JS, Ramiro S, et al. European League Against Rheumatism (EULAR) recommendations for the management of psoriatic arthritis with pharmacological therapies: 2015 update. Ann Rheum Dis 2016;75:499-510.

6. Harnett J, Wiederkehr D, Gerber R, et al. Real-world evaluation of TNF-inhibitor utilization in rheumatoid arthritis. J Med Econ 2016;19:101-12.

7. Hetland ML, Christensen IJ, Tarp U, et al. Direct comparison of treatment responses, remission rates, and drug adherence in patients with rheumatoid arthritis treated with adalimumab, etanercept, or infliximab: results from eight years of surveillance of clinical practice in the nationwide Danish DANBIO registry. Arthritis Rheum 2010;62:22-32.

8. Isaacs JD, Ferraccioli G. The need for personalised medicine for rheumatoid arthritis. Ann Rheum Dis 2011;70:4-7.

9. Biomarkers Definitions Working Group. Biomarkers and surrogate endpoints: preferred definitions and conceptual framework. Clin Pharmacol Ther 2001;69:89-95.

10. Strimbu K, Tavel JA, a TJ. What are biomarkers? Curr Opin HIV AIDS 2010;5:463-6.

11. Mohan C, Assassi S. Biomarkers in rheumatic diseases: how can they facilitate diagnosis and assessment of disease activity? BMJ 2015;351:h5079. 
12. Churov AV, Oleinik EK, Knip M. MicroRNAs in rheumatoid arthritis: altered expression and diagnostic potential. Autoimmun Rev 2015;14:1029-37.

13. Altman DG, McShane LM, Sauerbrei W, et al. Reporting Recommendations for Tumor Marker Prognostic Studies (REMARK): explanation and elaboration. PLoS Med 2012;9:e1001216.

14. Hayes DF. Biomarker validation and testing. Mol Oncol 2015;9:960-6.

15. Saad MN, Mabrouk MS, Eldeib AM, et al. Identification of rheumatoid arthritis biomarkers based on single nucleotide polymorphisms and haplotype blocks: a systematic review and meta-analysis. J Adv Res 2016;7:1-16.

16. Chemin K, Klareskog L, Malmström V. Is rheumatoid arthritis an autoimmune disease? Curr Opin Rheumatol 2016;28:181-8

17. Hwang SH, Jung SH, Lee S, et al. Leukocyte-specific protein 1 regulates T-cell migration in rheumatoid arthritis. Proc Natl Acad Sci U S A 2015;112:E6535-43.

18. Lee YH, Bae SC, Seo YH, et al. Association between FCGR3B copy number variations and susceptibility to autoimmune diseases: a meta-analysis. Inflamm Res 2015;64:983-91.

19. Murata K, Yoshitomi H, Tanida S, et al. Plasma and synovial fluid microRNAs as potential biomarkers of rheumatoid arthritis and osteoarthritis. Arthritis Res Ther 2010;12:R86.

20. Vicente R, Noël D, Pers YM, et al. Deregulation and therapeutic potential of microRNAs in arthritic diseases. Nat Rev Rheumatol 2016;12:496

21. Chen JQ, Papp G, Szodoray P, et al. The role of microRNAs in the pathogenesis of autoimmune diseases. Autoimmun Rev 2016;15:1171-80.

22. Kruse Meyer M, Andersen M. Effect of IL-6R inhibition with tocilizumab on the proteome of peripheral blood mononuclear cells from a rheumatoid arthritis patient. J Proteomics Bioinform 2015;8.

23. Bennike TB, Ellingsen $\mathrm{T}$, Glerup $\mathrm{H}$, et al. Proteome analysis of rheumatoid arthritis gut mucosa. J Proteome Res 2017;16:346-54.

24. Ortea I, Roschitzki B, López-Rodríguez R, et al. Independent candidate serum protein biomarkers of response to adalimumab and to infliximab in rheumatoid arthritis: an exploratory study. PLOS One 2016;11:e0153140.

25. Ortea I, Roschitzki B, Ovalles JG, et al. Discovery of serum proteomic biomarkers for prediction of response to infliximab (a monoclonal anti-TNF antibody) treatment in rheumatoid arthritis: an exploratory analysis. J Proteomics 2012;77:372-82.

26. Guma M, Tiziani S, Firestein GS. Metabolomics in rheumatic diseases: desperately seeking biomarkers. Nat Rev Rheumatol 2016;12:269-81.

27. Aletaha D, Neogi T, Silman AJ, et al. 2010 Rheumatoid arthritis classification criteria: an American College of Rheumatology/ European League Against Rheumatism collaborative initiative. Arthritis Rheum 2010;62:2569-81.
28. Rudwaleit M, Taylor WJ. Classification criteria for psoriatic arthritis and ankylosing spondylitis/axial spondyloarthritis. Best Pract Res Clin Rheumatol 2010;24:589-604.

29. Sieper J, Rudwaleit M, Baraliakos X, et al. The Assessment of SpondyloArthritis international Society (ASAS) handbook: a guide to assess spondyloarthritis. Ann Rheum Dis 2009;68(Suppl 2):ii1-ii44.

30. DANBIO. Dansk reumatologisk database. www.danbio-online.dk (accessed 4 Jul 2017).

31. Danish Regions. Bio- and Genome Bank Denmark. http://www. regioner.dk/rbgben (accessed 4 Jul 2017).

32. Kringelbach TM, Glintborg B, Johansen J, et al. The nationwide Danish Rheumatologic Biobank - paving the road to personalized treatment of patients with inflammatory rheumatic diseases. Eur Leag Against Rheum 2016.

33. Vandenbroucke JP, von Elm E, Altman DG, et al Strengthening the Reporting of Observational Studies in Epidemiology (STROBE): explanation and elaboration. Int J Surg 2014;12:1500-24.

34. Ibfelt EH, Jensen DV, Hetland ML. The Danish nationwide clinical register for patients with rheumatoid arthritis: DANBIO. Clin Epidemiol 2016;8:737-42.

35. Glintborg B, Sørensen IJ, Loft AG, et al. A nationwide non-medical switch from originator infliximab to biosimilar CT-P13 in 802 patients with inflammatory arthritis: 1-year clinical outcomes from the DANBIO registry. Ann Rheum Dis 2017;76:1426-31.

36. Glintborg B, Kringelbach T, Bolstad N, et al. Drug concentrations and anti-drug antibodies during treatment with biosimilar infliximab (CTP13) in routine care. Scand J Rheumatol 2018:1-4.

37. Hetland ML, Jensen DV, Krogh NS. Monitoring patients with rheumatoid arthritis in routine care: experiences from a treat-totarget strategy using the DANBIO registry. Clin Exp Rheumatol 2014;32:S141-6.

38. Li W, Sasso EH, van der Helm-van Mil AH, et al. Relationship of multi-biomarker disease activity score and other risk factors with radiographic progression in an observational study of patients with rheumatoid arthritis. Rheumatology 2016;55:357-66

39. Meyer GJ. Guidelines for reporting information in studies of diagnostic test accuracy: the STARD initiative. J Pers Assess 2003;81:191-3

40. Glintborg B, Kringelbach TM, Høgdall EVS, et al. Non-medical switch from originator to biosimilar infliximab in patients with inflammatory arthritis - impact on s-infliximab and antidrugantibodies. Results from the Danish Rheumatologic Biobank and the DANBIO Registry [abstract number 1997]. Arthritis Rheumatol 2016;68:270-6.

41. Haroon M, Winchester R, Giles JT, et al. Clinical and genetic associations of radiographic sacroiliitis and its different patterns in psoriatic arthritis. Clin Exp Rheumatol 2017;35:270-6.

42. DANBIO. National årsrapport 2015, 2016. 